DOI: 10.1002/ ((please add manuscript number))

Article type: Full Paper,

\title{
Novel applications of nonwood cellulose for blood typing assays.
}

Prof. Jasmina. Casals-Terré*1 ${ }^{1}$ Josep Farré-Lladós ${ }^{1}$, Allinson. Zuñiga ${ }^{1,2}$, Prof. Maria Blanca Roncero $^{2,}$ Prof Teresa Vidal $^{2}$

${ }^{1}$ Technical University of Catalonia, Mechanical Engineering Department, MicroTech Lab,Terrassa, Spain

2 Technical University of Catalonia, CELBIOTECH Paper Engineering Research Group, Terrassa, Spain

* Corresponding Author: Address: MicroTech Laboratory, Department of Mechanical Engineering, Technical University of Catalonia, Terrassa 08222, Spain. E-mail. Jasmina.casals@upc.edu

Keywords: Sisal-based paper, Lab-on-a-Chip, sisal, paper-based microfluidics, blood typing test, Point-of-Care Testing (POCT).

\begin{abstract}
Paper-based microfluidics devices can create a new healthcare model. Cellulose is carbohydrate polymer biocompatible and hydrophilic. These characteristics enhance the development of userfriendly diagnostic devices, but the link between paper manufacturing process and performance of the devices is still unclear. Previous studies focused on either commercial papers or lab papers from wood-cellulose fibers, with different basis-weight. This work introduces the effect of refining process and lab paper from non-wood-cellulose fibers, focusing on sisal fibers to overcome the aforementioned challenge. Structural characteristics of paper, such as basis-weight and degree of refining, are optimized and correlated with blood typing test resolution. Unrefined sisal paper of 50 $\mathrm{g} / \mathrm{m}^{2}$ and $100 \mathrm{~g} / \mathrm{m}^{2}$ basis-weight exhibit a higher gray intensity level than refined paper, and also maximal capillary rise and a pore size suitable for blood grouping tests. Two different blood types were evaluated with results consistent with the traditional methods, testifying the usefulness of this methodology.
\end{abstract}

\section{Introduction}

Paper is a well-established substrate for developing diagnostic devices since it is a 3D porous, lowcost, hydrophilic and biocompatible material. Paper strips were the earliest commercial uses of 
paper by the medical diagnostics industry. The World Health Organization (WHO) emphasized a need to further develop point-of-care devices which should be Affordable, Sensitive, Specific, Userfriendly, Rapid and Robust, Equipment-free, Deliver to the users who need them (ASSURED).

In 2007, Whitesides' Group introduced the use of techniques such as soft lithography to extend the advantages of microfluidic technology to paper substrates ${ }^{[1][2] ~[3] ~ a n d ~ t h e r e f o r e, ~ i m p r o v i n g ~ t h e ~}$ Affordable, Sensitive, Specific and Rapid (ASSR) capabilities of paper based sensors. Low volumes, low energy consumption, high sensitivity and fast turnaround of test results are properties deeply appreciated in economies with limited resources thanks to the use of paper as a substrate. [4] [5] [6] Due to the fact that paper is a $3 \mathrm{D}$ complex porous structure, a better understanding of its physicochemical properties is needed to improve the User-friendly, Equipment-free and Deliver to the users who need them (UED) characteristics of POC devices, as pointed in the review of Nilghaz et al. ${ }^{[7]}$

Paper characteristics such as different wood fiber types, porosity and thickness for lab paper and commercial papers showed its impact on the easiness of the test readout for the end user during a blood typing test. ${ }^{[8]}[9]$. However, the refining effect and the use of non-wood fibers has not been studied before. The refining is a common mechanical process used in paper manufacturing, which can change the paper network, and therefore can affect the efficiency of the blood test. Initially researchers focused on chromatography and Whatman filter papers. However, its high complexity promoted the investigation of homemade paper to focus on the effects of paper structure (pore size, thickness and fiber type ${ }^{[8][9]}$ and the use of other cellulose derivates commonly used in lateral flow assays such as nitrocellulose ${ }^{[10] .}$ Recently, novel technologies such as 3D-printing have allowed researchers to reproduce a regular well-known paper network and tune the artificial paper properties more accurately to study the effects on blood motion ${ }^{[11]}$. The porous structure of threads motivated their application in a similar approach to perform low cost blood typing test, initially using polyester threads ${ }^{[12][13]}$. However, there is an undeniable growing interest in using natural renewable resources. This translated in the use of silk threads for blood typing [14] . Another 
renewable resource are non-wood plants at present, basically because they can be obtained in large amounts from agricultural residues that are rich in cellulose fiber and because the originating plants can be grown in annual cycles. In some geographical areas, these materials constitute the main or even only source of fiber ${ }^{[15]}$. Paper in any grade can be manufactured by pulping appropriate mixtures of non-wood fibers. Aracri et al. found sisal (Agave sisalana) to contain a high proportion of cellulose, with similar physical characteristics to the ones proposed as optimal for wood-based papers ${ }^{[16]}$. In this paper, we investigated the effect of paper structure on a novel paper substrate obtained from sisal fibers to perform blood typing tests.

Blood typing tests are extensively studied since they are required in non-programmed medical situations in order to prevent patients' so-called “transfusion reaction” occurring immediately upon transfusion of incompatible blood and causing severe health problems or death. The most important blood group is $\mathrm{ABO}$, defined in terms of $\mathrm{B}$ and $\mathrm{A}$ antigens on the surface of the red blood cells, and their corresponding isoagglutinins. For examples, blood group A is characterized by A antigen on the red cells and anti-B isoagglutinin in the plasma; blood group $\mathrm{O}$ is characterized by the absence of $\mathrm{A}$ and $\mathrm{B}$ antigens on red cells, and both anti-A and anti-B isoagglutinins in the plasma. Another blood group antigen of great importance is the $\mathrm{D}$ antigen of the Rh blood group system. ABO and $\mathrm{Rh}(\mathrm{D})$ antigen typing are routinely performed for pre-transfusion and pre-natal patients. Typing of red cells is based on the hemo-agglutination reaction, using commercial anti-A, anti-B and anti-D antisera.

In this work, we characterize a novel non-wood-cellulose paper derived from a highly renewable resource, and explore its potential for blood typing tests.

\section{Materials and methods}

This section describes the methodologies used to develop a blood typing test on novel sisal-based paper.

\subsection{Lab sisal paper at different refining degree and Whatman filter papers}

\subsubsection{Lab sisal paper}


In order to produce lab sisal paper, elemental chlorine free (ECF) bleached sisal pulp was purchased from CELESA mill in Tortosa (Spain). According to ISO5263 the pulp was initially disintegrated at 30,000 revolutions, and then, in a PFI mill, three aliquots of pulp were refined at 0, 2000 or 6000 revolutions per minute following ISO 5264. The refining process is a mechanical treatment that modifies the structure of fiber surface and increases interfiber bonding capacity, modifying the paper network depending on the refining degree. Thus, enhancing the sheet formation and its mechanical properties. After the refining, the samples were disintegrated for 10,000 rev to determine Schopper-Riegler degree ( ${ }^{\circ} \mathrm{SR}$ ) and the water retention value (WRV) according to ISO 5267 and ISO 23714, respectively. Fiber length and percentage of fines and width were measured according to TAPPI standard method T271 using a Kajaani FS300 fiber analyzer. Rapid-Köthen method according to ISO 5269 was used to prepare sheets of two different basis weight (50 or 100 $\mathrm{g} / \mathrm{m} 2)$ from each refined pulp.

\subsubsection{Whatman Paper}

Whatman filter papers $(\# 5,40,1,41)$ were purchased from Fisher Scientific.

\subsection{Paper properties characterization}

Before physical characterization, the papers were conditioned for at least $24 \mathrm{~h}$ hours at $23^{\circ} \mathrm{C}$ and 50\% relative humidity, according to ISO187. Basis weight, thickness and apparent density, and air permeability by Bendtsen method properties were measured from ten replicates according to ISO 536, ISO 534 and ISO 5636-3 standards, respectively. Capillary rise was measured by the Klemm method (ISO 8787), where a strip of paper is suspended and partially immersed in water, and it is measured the distance that the water rise in $10 \mathrm{~min}$. It was performed 4 replicates for each sample of paper. The pore size distribution was also measured by a liquid displacement method with the Porometer 3G analyzer (Quantachrome Instruments) according to ASTM F316-03 standard. The Grubbs’ Test was applied to detect outliers at a 0.05 significance level. Experimental errors were calculated as mean and standard deviation in accordance with the respective standards. Table I 
shows the nomenclature used. These properties were also measured on Whatman papers, except the average pore size which is the nominal particle retention size provided by the manufacturer.

Surface morphology of papers was evaluated by taking surface SEM pictures on a JEOL JSM-6400 Scanning Electron Microscope, at different degrees of magnification and voltages (5-15kV).

\subsection{Pattern printing on paper}

PRUSA i3 Printer with a blunt G25 needle injector was used to deposit and design the cross-pattern using a hydrophobic mixture of alkylketene dimer (AKD) and colophony (Fennosize G7020F purchased from KERIMA) ${ }^{[17]}$ This product requires a thermal curing to achieve the hydrophobicity, therefore, the printed patterns were heated in an oven at $100^{\circ} \mathrm{C}$ for $10 \mathrm{~min}$ just after printing.

\subsection{Blood and antibodies}

Blood was obtained from healthy donors and stored in ethylenediamine tetra-acetic acid (EDTA) tubes at $4^{\circ} \mathrm{C}$ less than 2 days before tested. Blood was initially characterized using conventional methods in Laboratori de Referencia de Catalunya.

Analytical grade $0.9 \% \mathrm{NaCl}$ saline solution and phosphate buffered saline (PBS) were purchased from Sigma Adrich. DiaClon Monoclonal IgM antibodies Anti-A, Anti-B and Anti-D for blood grouping characterization were purchased from Bio-Rad.

\subsection{Methods for blood typing test}

Forward blood typing test was implemented on a cross-pattern. Three of the ends of the cross allocated the required antibodies (anti-A, Anti-B and anti-D are deposited) and one end acted as a control region. The use of a cross avoids the need of multiple blood sample depositions and the advantage of a differential readout of the results thanks to the control zone, which minimizes the effects of different lightning conditions.

Figure 1 shows the testing procedure for blood typing. Initially, 15-30 $\mu \mathrm{L}$ fresh blood were diluted 1:1 with 15-30 $\mu \mathrm{L}$ PBS and deposited in the center. Previously, $1.5 \mu \mathrm{L}$ of DiaClon Monoclonal IgM antibodies Anti-A, Anti-B and Anti-D from Bio-Rad had been pipetted onto each reaction area. The pattern was then gently rinsed with $0.9 \% \mathrm{NaCl}$ saline solution. A picture was taken with a 
conventional cell-phone camera and the intensity of the blood spot was quantified by ImageJ software. The gray scale mean optical density of spot was compared on each region. Near the hydrophobic barrier, the hydrophobic material can shrink the average pore size, therefore the read out should always be done in the middle of the square. To standardize the measurement, a rectangle that covered $1 \mathrm{~mm}$ of the border was used to mark the reading area, see Figure 1 (e).

The relationship between mean optical intensity color in gray scale $30 \mathrm{~s}$ after washing a diluted droplet 1 blood: 1 PBS were investigated using identical lighting conditions. The grey intensity level value was measured to quantify the positive and negative result. A high grey intensity value means a clean area therefore a negative result. 


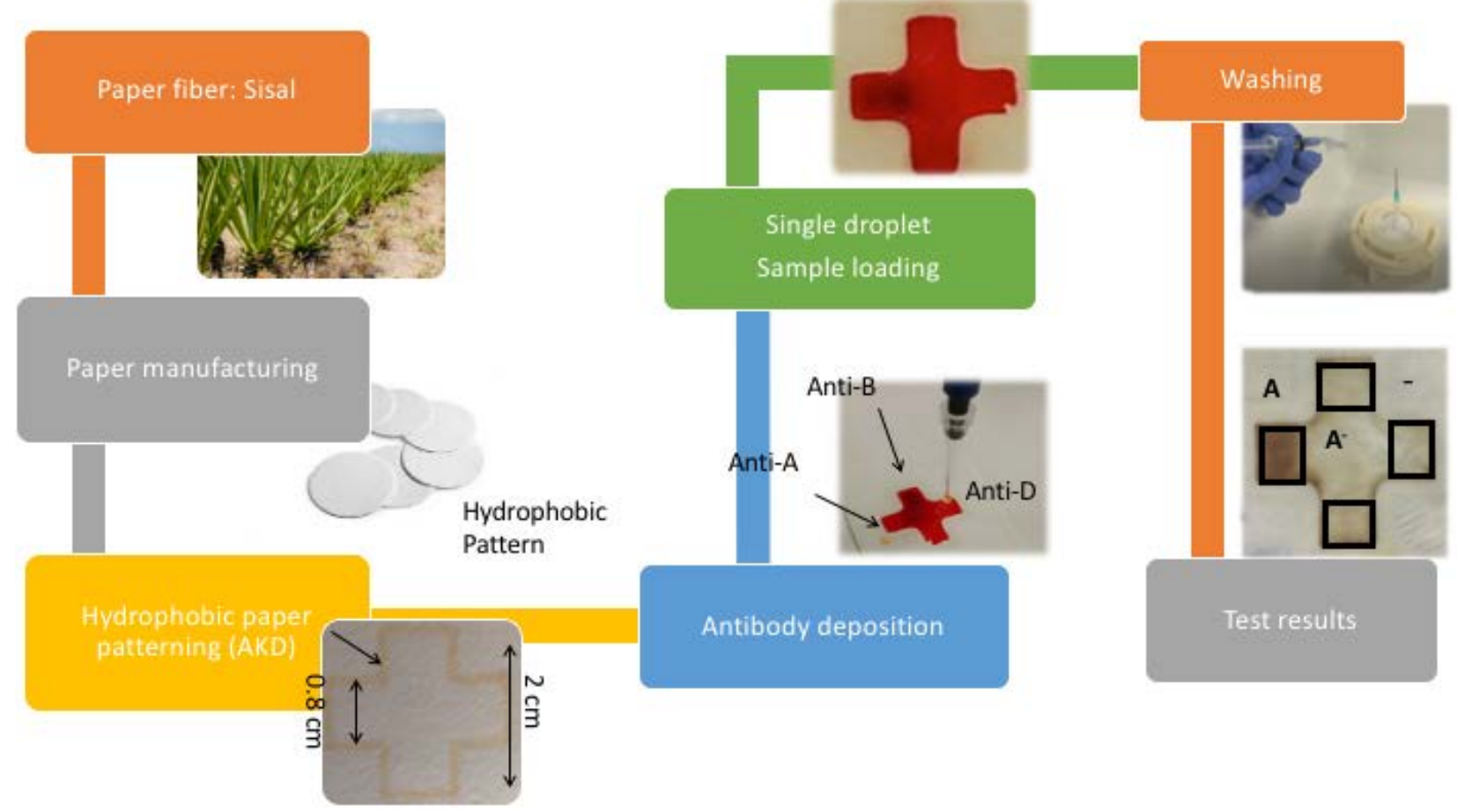

Figure 1 Schematics of (a) hydrophobic pattern for forward ABO blood typing. (b) Deposition of blood sample. (c) Deposition of antibodies. (d) Washing with saline solution.(e) Test results analysis

\section{Results}

Table I shows selected characteristics and properties of Whatman paper and hand sheets sisal based paper obtained in different basis weights by using also different degrees of refining (revolutions per minute).

\subsection{Effect of refining process on fiber morphological properties}

During the refining process, the ${ }^{\circ} \mathrm{SR}$ increased. ${ }^{\circ} \mathrm{SR}$ values are related with the conformability that is how the fibers conforms to the other fibers during the papermaking process. The higher the ${ }^{\circ} \mathrm{SR}$, the better the conformability, and therefore the fibers are more highly bonded on the paper. WRV value is also correlated to the bonding ability of fibers, and as it also increased during the refining process, a higher bonding between fibers could be predicted.

As can be seen in Figure 2 (a), fiber length decreased with refining and at the same time, the percentage of fines increased. Fiber shortening and fines formation are two common effects of 
refining of fibers. Fines are small fragments (less than $200 \mu \mathrm{m}$ ) removed from the external walls of fiber and, as they have a very large specific surface area, they improve bonding between fibers ${ }^{[18]}$. Prior works have documented the influence of fiber length (comparing hardwood and softwood fibers) in improving the quality of blood typing results ${ }^{[8] .}$ Su et al. found that papers from softwood (long) fibers were more efficient for blood typing ${ }^{[9]}$. However, Li et al. (2014) reported that hardwood (short) fibers gave rise to a paper with higher porosities than softwood fibers, allowing a better RBC transport and a better blood analysis test ${ }^{[8]}$. Fiber lengths of softwoods average between 2 and $6 \mathrm{~mm}$, and of hardwoods between 0.4 and 1.6; and fiber width is between 20 and $50 \mu \mathrm{m}$ in softwoods, and between 10 and $40 \mu \mathrm{m}$ in hardwoods ${ }^{[19] .}$ The length of the non-refined nonwood sisal fibers was $1.4 \mathrm{~mm}$ and the fiber width was $16.03 \mu \mathrm{m}$ (Table II), which is similar to the species of hardwood fibers with longer fibers.

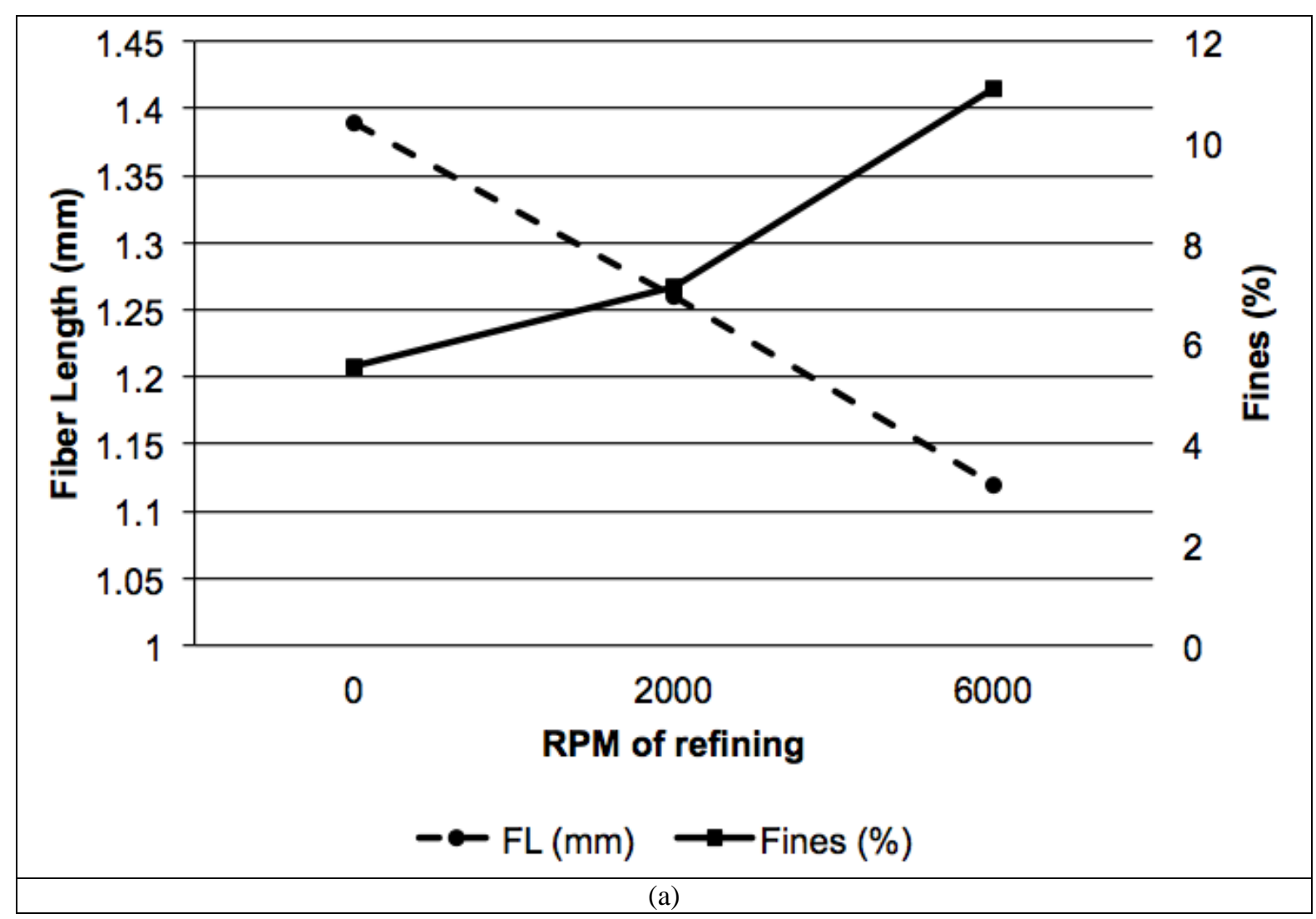




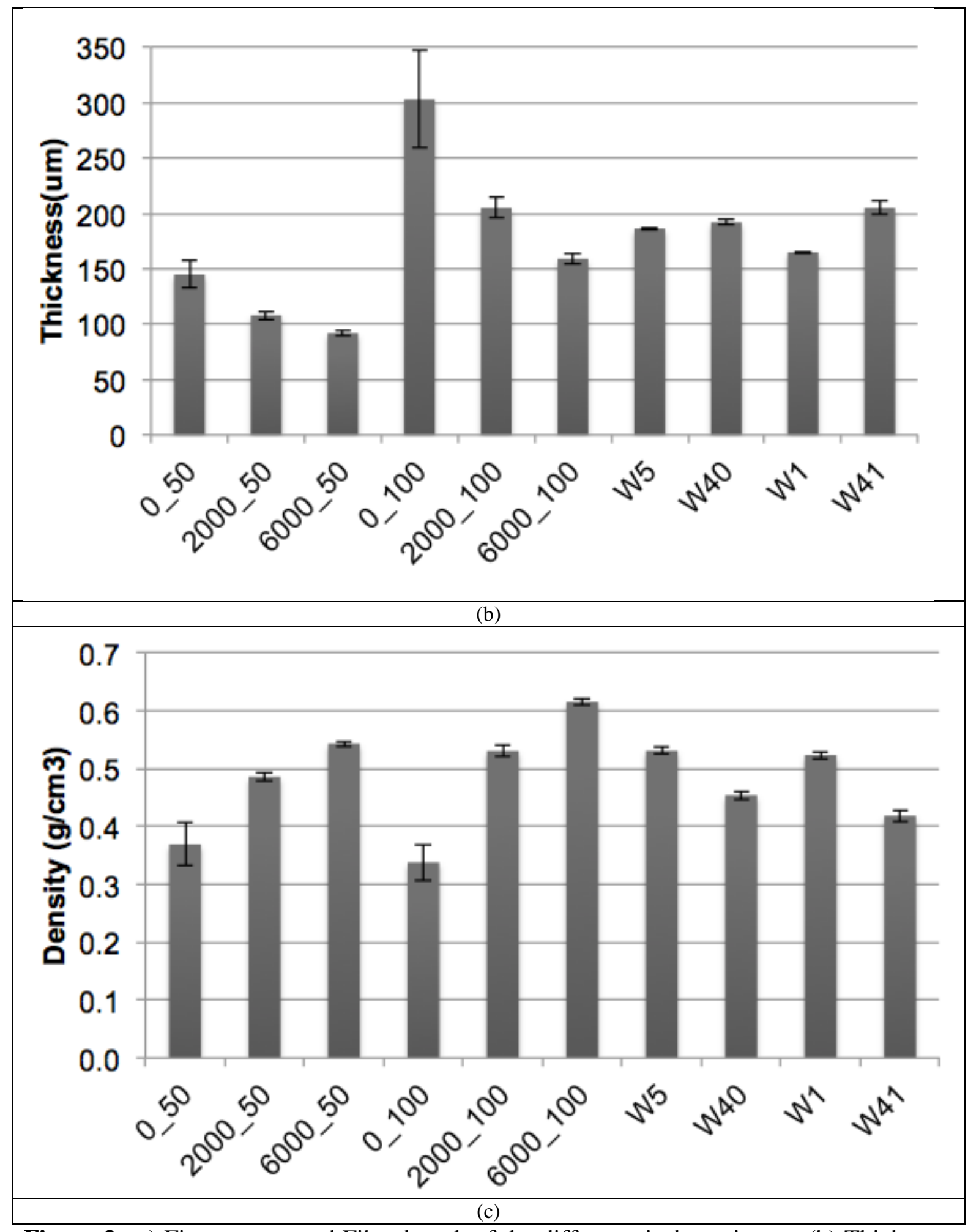

Figure 2. (a) Fine content and Fiber length of the different sisal specimens. (b) Thickness values of hand-sheets sisal papers (at different basis weight and refining degree) and of Whatman papers (c) Apparent density values of hand-sheets sisal papers (at different basis weight and refining degree) and Whatman papers 


\subsection{Structural properties of tested papers}

Thickness values of sisal sheets with $50 \mathrm{~g} / \mathrm{m}^{2}$ of basis weight were lower than that of $100 \mathrm{~g} / \mathrm{m}^{2}$. As can be seen in Figure 2 (b), this property decreased when the degree of refining was higher while the basis weight was kept constant. Internal and external fibrillation are two major effects of refining, which makes the fibers more flexible, conformable and with more specific surface area, contributing to increase the fiber bonding effect ${ }^{[20]}$. The refining process improved the bonding of fibers, leading to a densification of paper, as can be seen in Figure 2 (c), where the apparent density was increased during refining. This means that papers with high density had less quantity of vacancies (volume with no fiber coverage).

Concerning the commercial papers (Whatman papers \#5 and \#1), they had similar thickness and density values than sisal papers at $2000 \mathrm{rev}$ and $100 \mathrm{~g} / \mathrm{m}^{2}$. Therefore, these papers will be compared in the next properties.

A Scanning Electron Microscope SEM was used to observe the cellulosic fiber network of the different tested papers. In Figure 3 SEM images confirm the modification of surface fibers by the refining process. Non-refined sisal fibers (0_100 paper) show a uniform and smooth surface. Nevertheless, refined sisal fibers (2000_50 and 6000_100) are more roughness and show a surface delamination and a pilling off fibrils, which is known as external fibrillation. This effect is clearly manifested with the sample 6000_100 in Figure 3 (b) where it is shown some delaminated layers or fibrils that are still attached and are responsible of fiber roughness and also contribute to increase the specific surface area of fibrils, improving the fiber-fiber bonding. As a result, the conformability of fibers and the fiber network of paper is improved. This better conformability has an effect on the pore size distribution, as can be noticed in the Figure 3 (a), where a higher closed network structure is showed whenever the refining is extended.

Concerning Whatman paper, it looks like a refined paper, but not as much as 6000_100 sisal paper. The width of the sisal fibers is similar to the width of the fibers from Whatman papers. However, 
sisal fibers are straighter than that from Whatman paper, resulting in a more heterogeneous network structure in the Whatman paper, and therefore, in a less uniform pore distribution

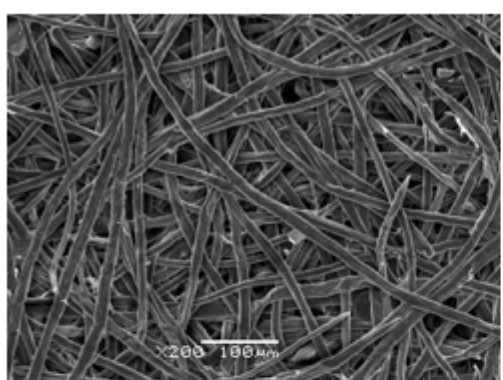

0_1000 rpm $100 \mathrm{~g} / \mathrm{m}^{2}$

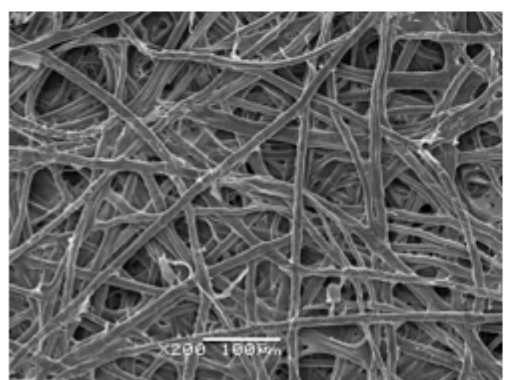

6000_1006000 rpm $100 \mathrm{~g} / \mathrm{m}^{2}$

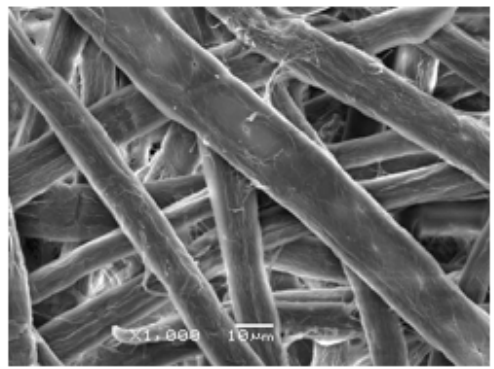

0_1000 rpm $100 \mathrm{~g} / \mathrm{m}^{2}$

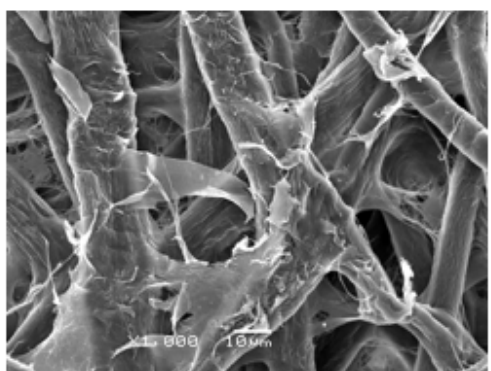

6000_1006000 rpm $100 \mathrm{~g} / \mathrm{m}^{2}$

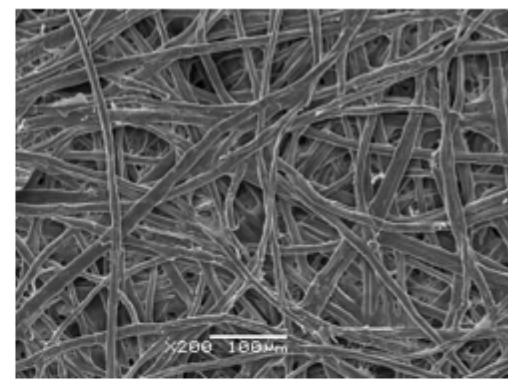

2000_50 2000 rpm $50 \mathrm{~g} / \mathrm{m}^{2}$

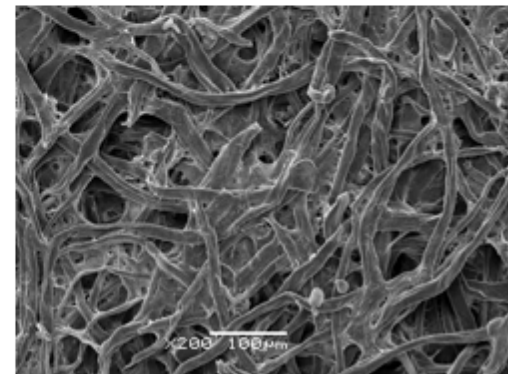

W 41 Whatman 41

(a)

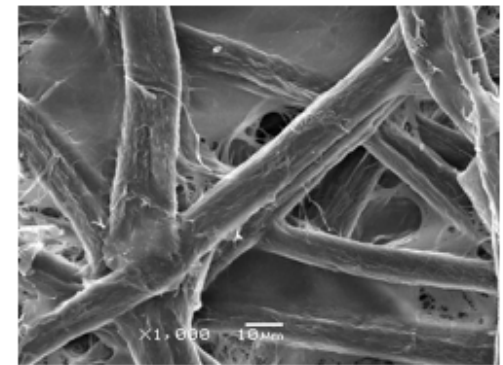

2000_50 $2000 \mathrm{rpm} 50 \mathrm{~g} / \mathrm{m}^{2}$

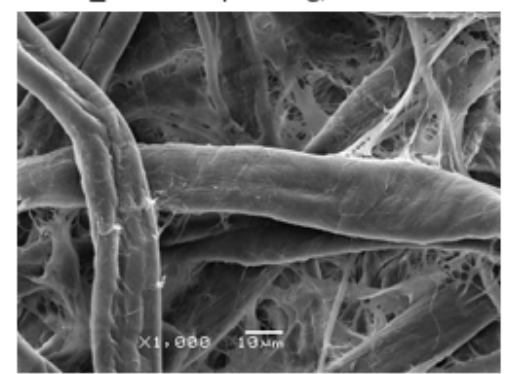

W 41 Whatman 41

(b)

Figure 3. SEM images of sisal papers and Whatman 41 at (a) $200 \mathrm{X}$ and (b) $1000 \mathrm{X}$ magnification. Sisal papers: 0_100 (0 rev and $100 \mathrm{~g} / \mathrm{m} 2), 2000 \_50\left(2000 \mathrm{rev}\right.$ and $\left.50 \mathrm{~g} / \mathrm{m}^{2}\right)$ and 6000_100 (6000 rev and $\left.100 \mathrm{~g} / \mathrm{m}^{2}\right)$ 


\section{WILEY-VCH}

\subsection{Pore size distribution of tested papers}

As can be seen in Figure 4(a), the pore size was higher in the case of lower basis weight sheets, indicating a more porous internal network, according with the density values (Figure 2 (c)) and also agreeing with the findings of $\mathrm{Li}$ et al. and Carstens et al. ${ }^{[8][21]}$ Moreover, the stronger the refining, the lower the pore size. This is also related with the higher flexibility of fibers reached during the refining process, enhancing the fiber bonding effect, which has been corroborated with the SEM images (Figure 3) ${ }^{[22] \text {. }}$

The profile of the pore size distribution of non-refined sheets (0_50 and 0_100) exhibits a shoulder, which disappear or decreases as well as the refining is stronger. The peak in the profile of refined sheets is sharper. This behavior suggests that the internal pore size distribution of the refined samples is more uniform, since poly-dispersion of the profile is lower.

The pore size distribution of Whatman papers are located below $20 \mu \mathrm{m}$, in the same range of refined sisal papers (Figure 4 (b)) and it has been also suggested from Figure 3. It can be seen that these papers have more irregular profiles, suggesting a less uniform internal network, due to a less straight fibers as it is shown in Figure 3. 
WILEY-VCH

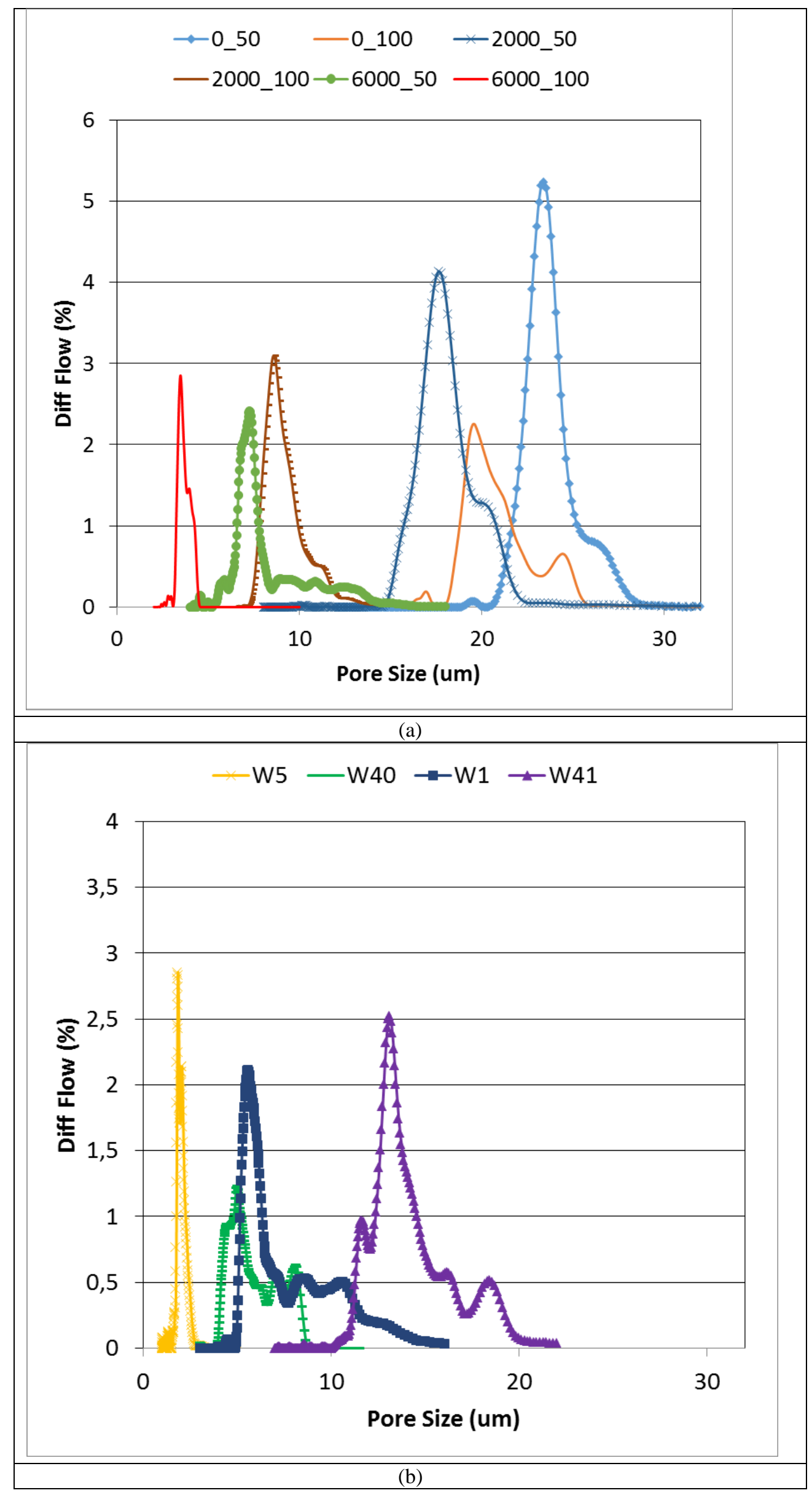




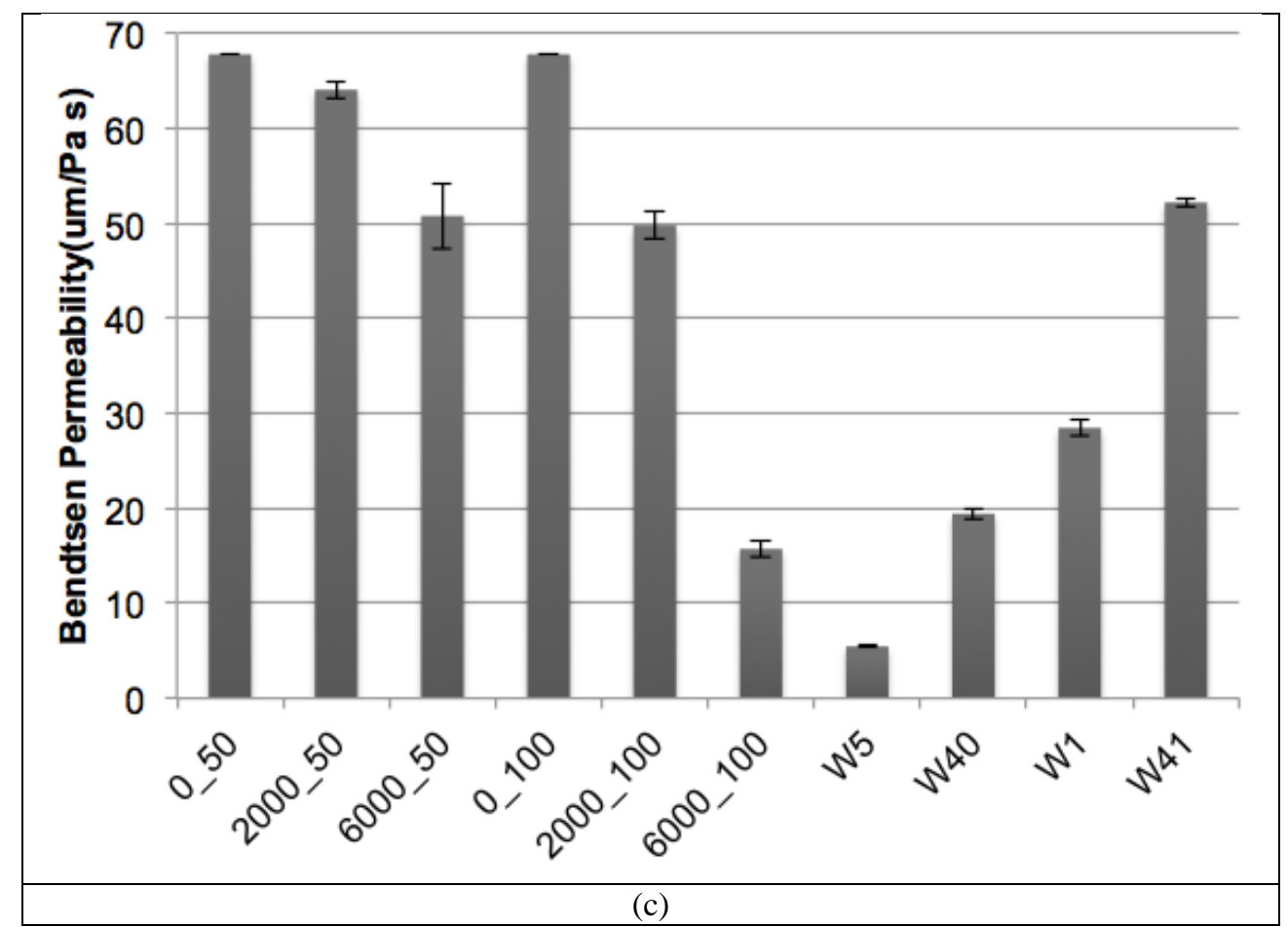

Figure 4. (a) Pore size distribution of hand-sheets sisal papers, at different basis weight and refining degree. (b) Pore size distribution of tested Whatman papers. (c) Air permeability by Bendtsen method of studied papers.

The behavior of pore size distribution in the studies papers correlates with the air permeability measurements, as can be seen in Figure 4(c).

\subsection{Influence of paper properties on cleaning efficiency}

User-friendly devices should provide a clear difference between positive and negative results. In the designed blood typing test, a positive result means that RBCs are agglutinated and trapped into the internal structure of paper which provides a low grey intensity level (the paper is stained), while a negative result means that RBCs do not agglutinate and are free to move by capillarity through the fiber network, therefore the paper is clean after washing and the grey intensity level value is high. Li et al. found that the positive tests were not significantly influenced by basis weight or fiber type, which was opposite in the case of negative tests ${ }^{[8] .}$ Comparing grey intensity levels from Figure 5 (a), in sisal specimens as the refining increase after the washing step the level of cleanness decreases. From Figure 2 (a), 


\section{WILEY-VCH}

we know that these papers have an increase of fines in their network. Li et al. pointed that since RBCs are negatively charged, their flow could be modified by fines accumulated between the fiber ${ }^{[8]}$. According to Figure 5 (a), fines decrease the mobility of RBC along the paper network. Moreover, an increase of basis weight also slightly decreases the clarity, which can be attributed to the fact that the paper is more complex and not all RBC follow the same flow path. In Whatman papers, which all have a similar basis weight $\left(100 \mathrm{~g} / \mathrm{m}^{2}\right)$, the mobility of RBCs is low and all the paper present a low clarity value, which could lead to false positive readout. In Whatman papers, even though they have different range of pore size which would expect different flow behavior of RBC we notice that the clarity value is low and similar to sisal 6000_100.

From the measured properties of the papers (Table II), capillary rise and pore size show a linear relationship with the grey intensity level after washing, see Figure 5 (b) and (c). Papers with high capillary rise values are cleaned better than those with low values. A similar behavior is observed when plotting the pore size. Whatman papers follow the same trend but they are always less clean even with similar pore sizes. The deviation between commercial Whatman papers and lab-sheet sisal papers can be due to additives used to enhance paper properties (such as wet strength additives), which have not been used in sisal papers, or it can be due to the less uniform pore distribution or the higher heterogeneous structure of Whatman paper (Figure 3). Based on these results, Whatman papers and sisal specimen 6000_100 were excluded to implement the complete test to avoid low specificity in the results. 


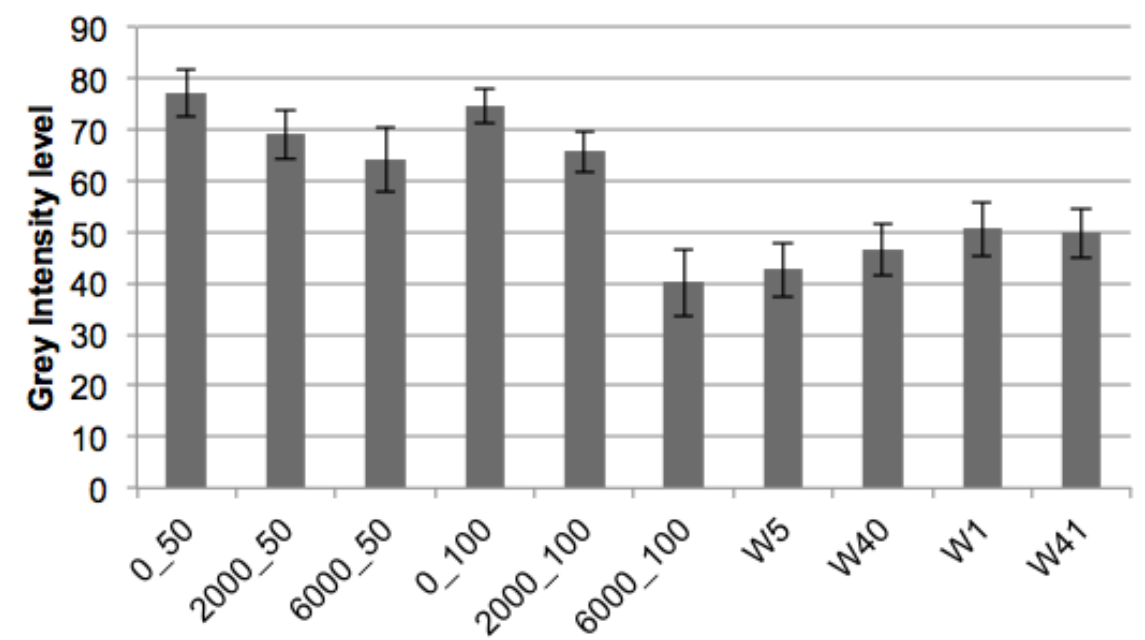

(a)

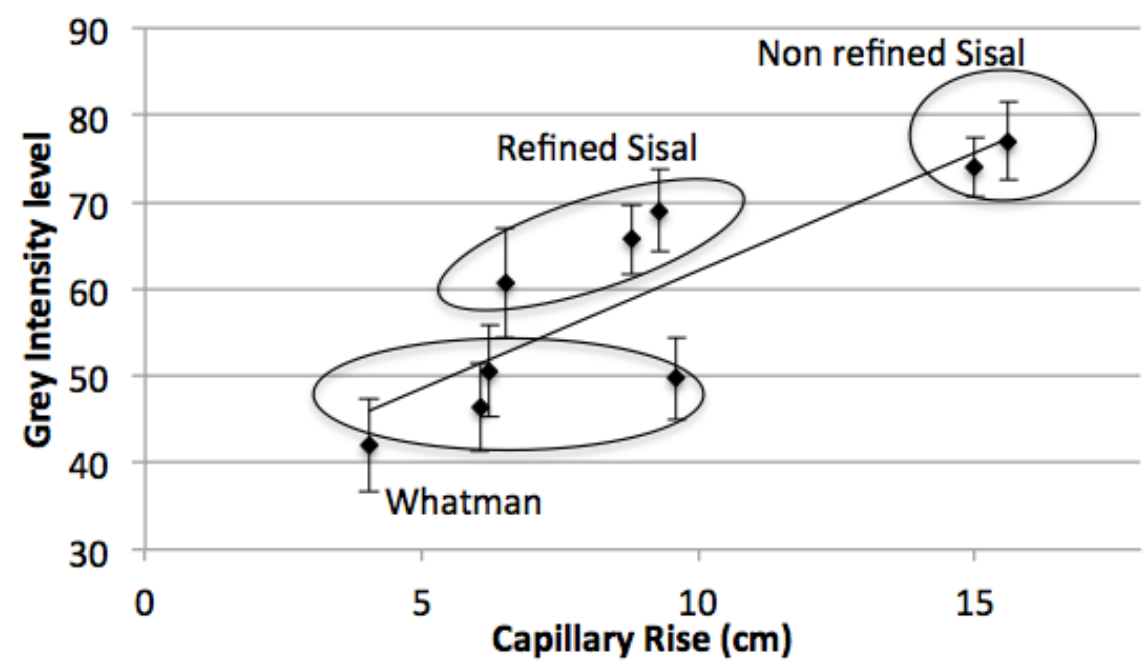

(b)

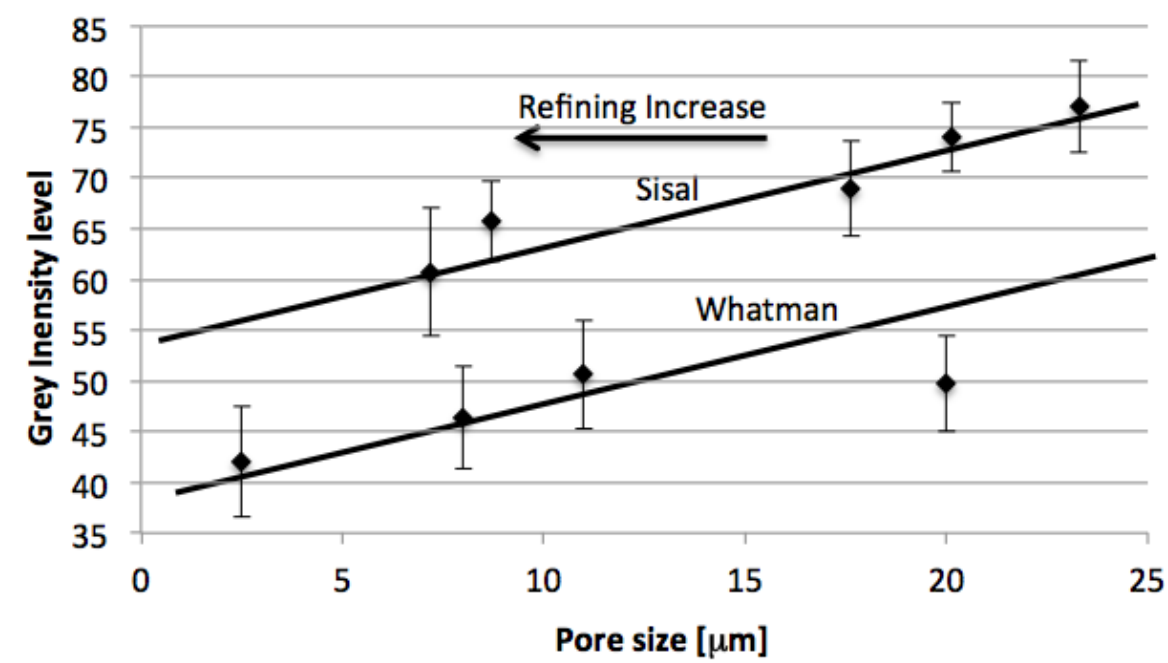

(c)

Figure 5. (a) Grey intensity level after washing a droplet of diluted blood:PBS (1:1). Grey intensity levels of sisal and Whatman paper after cleaning in blood:PBS (1:1) as a function of (b) capillary rise and (c) pore size. 


\section{WILEY-VCH}

\subsection{ABO blood typing tests}

Two different blood types: A- and O- were characterized from different healthy volunteers after being characterized by conventional methods. It was used the cross-pattern designed in this research (Figure 1). Supplementary material shows test results on other blood types $\mathrm{A}+, \mathrm{B}+$ and $\mathrm{O}+$. In $\mathrm{A}-$ blood type, the area where Antibody $\mathrm{A}$ had been previously deposited should hemoagglutinate and prevent RBCs from moving decreasing the grey intensity level. In case of O- blood, all the areas should be cleared after saline solution washing, giving rise to higher grey intensity level values. The test was done on five different sisal paper specimens and Figure 6 (a) shows an example of a test results real picture on each Apaper specimen. The paper specimens subjected to strong refining (6000_50 and 2000_100) exhibited less sensitivity.

Pore sizes and density of the papers were investigated to understand which paper could provide a higher relative grey intensity level (difference between positive and negative value). Following the same trend shown by negative results pore size is an important factor for discrimination, Figure 6 (b) shows that papers with pore sizes between $15 \mu \mathrm{m}$ and $25 \mu \mathrm{m}$ are the ones achieving higher relative value between positive and negative results. These pore size values are around two times the RBC diameter (which normally range between 7 to 8 microns). If an agglutination of two or more RBC is formed, it will not be able to flow easily, while a non-agglutinated RBC can move without problems. Figure 6 (c) depicts a decrease of discrimination between positive and negative results when paper density is increased. This can be due to an increase on fiber network complexity dificulting the free movement of nonagglutinated RBCs. 
WILEY-VCH

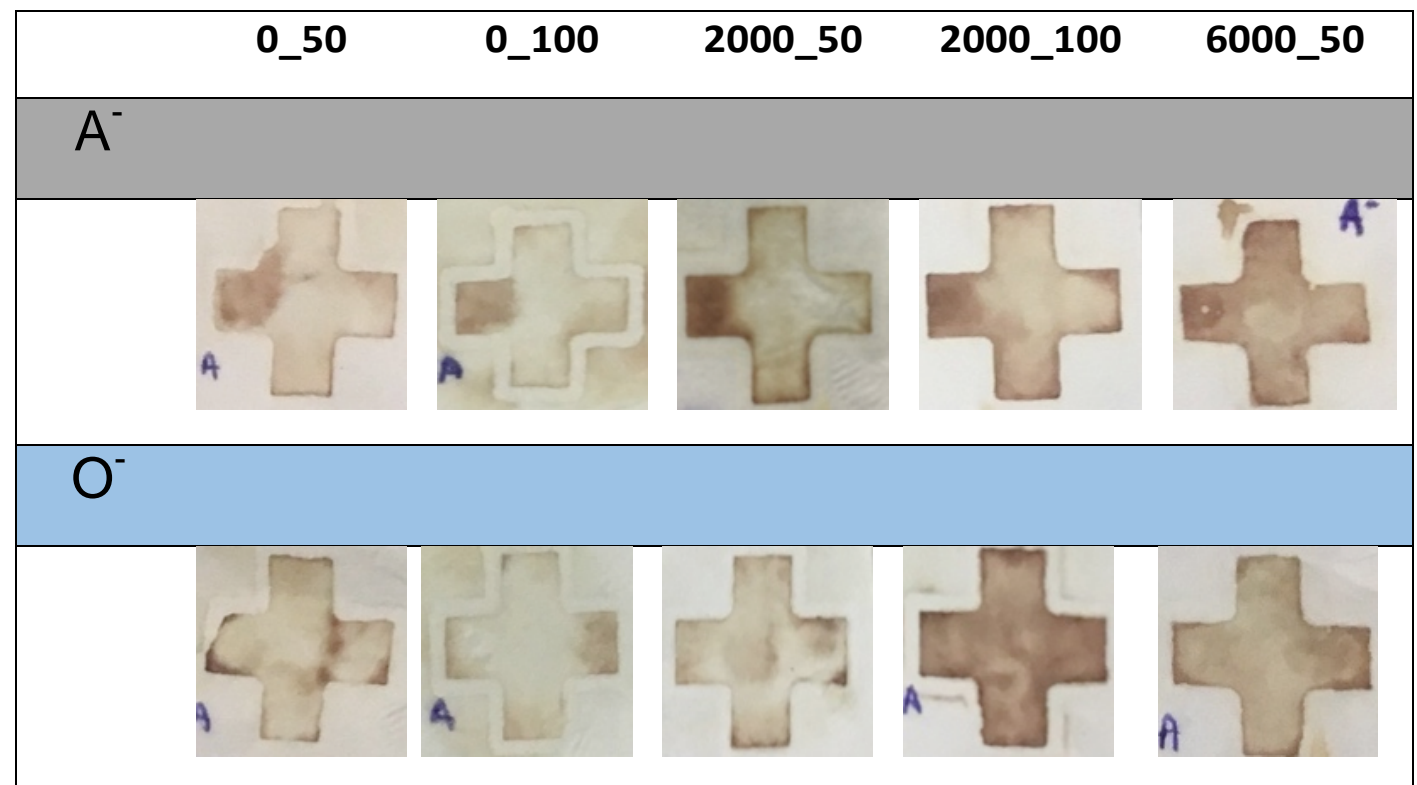

(a)

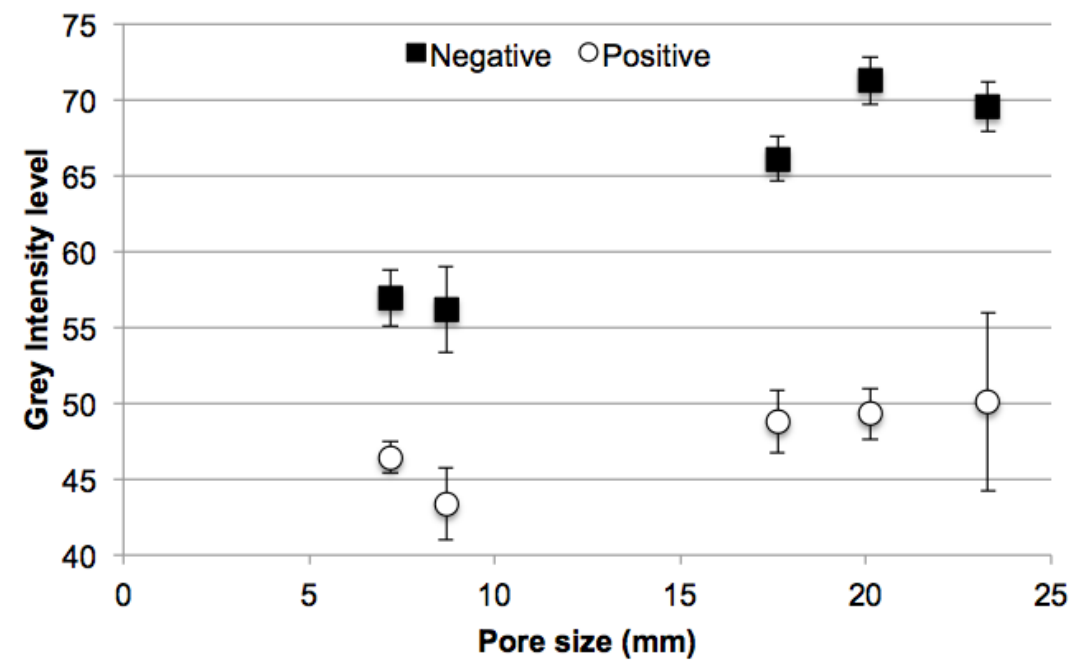

(b)

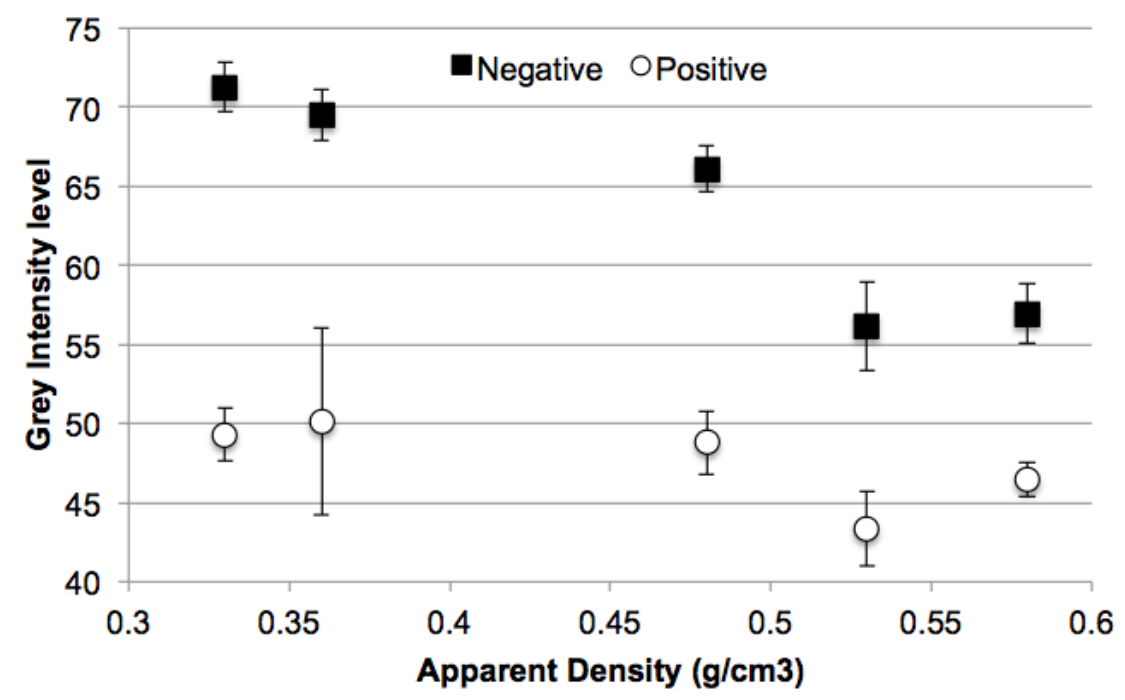

(c)

Figure 6 (a) ABO blood typing with microfluidic sisal based paper. Positive and negative grey intensity levels of sisal papers as a function of (b) pore size and (c) apparent density. 
To our knowledge, this is the first study to have investigated the effectiveness of sisal based paper for designing Forward ABO blood tests. The results testify to the potential of sisal paper for developing low cost point-of-care tests.

\section{Discussion}

Microfluidic researchers have mainly proposed two different approaches to tackle the challenge of producing paper-based blood typing tests: elution type tests ${ }^{[23][24][25]}$ and flowthrough tests ${ }^{[6][26] .}$ Elution tests have been used for weak blood group detection, such as minor blood groups and reverse blood grouping ${ }^{[26][27] .}$ A reliable blood typing device must provide to the end user a clear result to differentiate without doubt a positive result from a negative result. In this test, a positive result is caused by hemagglutinated red blood cells trapped in the fiber network of the paper providing a red dot. On the other hand, a negative result shows a clear area since non-agglutinated red blood cell are washed away during the rinsing step of the test. Hemagglutination is reversible and in weak blood group readout differentiation between positive and negative results is sensitive to the forces applied during the washing step that can break apart some initial agglutinates. On the contrary, flow-through tests can provide faster readout results, thus is used for forward blood typing usually which generally create stronger agglutinates $^{[6][28]}$

There is no doubt that anisotropic characteristics of paper influence on the RBC movements along the fiber network and the need of a growing interest to gain in understanding. Numerous investigations have tackle this need changing to more controlled materials, such as $3 D$ printed artificial paper, cellulose derivate or threads with similar results ${ }^{[7][10]-[14]}$

Initial works focused on chromatography and Whatman filter papers, which have special properties (e.g., uniform pore size and permeability, high wet strength, low ash content, and acid and oil resistance) that are dependent on the intended application ${ }^{[28] .}$ However, its high 


\section{WILEY-VCH}

complexity promoted the investigation of homemade paper to focus on the effects of paper structure (pore size, thickness and fiber type) ${ }^{[8][9] .}$

There is an undeniable growing interest in using natural renewable resources such as nonwood plants at present.

In this paper, we investigated the effect of paper structure on a novel paper substrate obtained from sisal fibers. Lab papers with different basis weights and degree of refining were made without any added chemical additive. The influence of the paper manufacturing conditions (basis weight and degree of refining) was examined with a view to obtaining sisal paper with different structural characteristics and assessing their impact on flow through blood typing results and compared to commercial Whatman filter paper. The paper was optimized for a clear distinction between agglutination of RBCs by effect of antibody-antigen interactions and free to move RBCs. An AKD printed microfluidic cross pattern was used to implement a single step blood typing test on lab sisal paper.

\section{Conclusions}

In this work, we physically characterized sisal paper to assess the fitness of this novel paper for developing forward ABO blood typing tests. The unrefined paper specimens at different basis weight (0_50 and 0_100) and the slightly refined (2000_50) had a pore size greater than $15 \mu \mathrm{m}$, low density and low \% of fines and provided blood typing results fully consistent with those of the conventional tests. Therefore, physical properties and preliminary studies with two blood types suggest that the sisal paper can be further studied for paper-based blood typing. Further studies will consider the effect of wet strength agents on this type of blood typing test and the use of sisal paper in different diagnostic approaches.

This truly low-cost, straightforward manufacturing medium from a widely-available, renewable resource thus constitutes a major addition to the existing array of paper production 


\section{WILEY-VCH}

devices for point-of-care bio-applications, especially in remote regions and resource-limited environments (e.g., small laboratories, private clinics).

\section{Acknowledgements.}

The research for this paper was financially supported by the Spanish Ministry of Economy and Competitivy, grant no. CTQ2013-48995-C2-1-R, CTQ2016-77936-R (funding also from FEDER) and CTQ2017-84966-C2-1-R. The authors also thank Gloria Soria and Anna Padrós of the Laboratori Referencia de Catalunya for technical advice in assay implementation.

\section{Ethics.}

Universitat Politècnica de Catalunya ethical committee approved the tests performed during this study.

\section{References}

[1] A. W. Martinez, S. T. Phillips, M. J. Butte, and G. M. Whitesides, "Patterned Paper as a Platform for Inexpensive, Low-Volume, Portable Bioassays,” Angew. Chemie Int. Ed., vol. 46, no. 8, pp. 1318-1320, Feb. 2007.

[2] D. A. Bruzewicz, M. Reches, and G. M. Whitesides, "Low-Cost Printing of Poly(dimethylsiloxane) Barriers To Define Microchannels in Paper,” Anal. Chem., vol. 80, no. 9, pp. 3387-3392, May 2008.

[3] A. W. Martinez, S. T. Phillips, B. J. Wiley, M. Gupta, and G. M. Whitesides, "FLASH: A rapid method for prototyping paper-based microfluidic devices,” Lab Chip, vol. 8, no. 12, p. 2146, Dec. 2008.

[4] C. Tao, C.-S. Yen, J.-T. Liu, and C.-J. Chen, "Analytical performance of paper electrobiosensor detection platform for point-of-care diagnosis,” Cellulose, vol. 23, no. 6, pp. 3799-3808, Dec. 2016.

[5] A. W. Martinez, S. T. Phillips, E. Carrilho, S. W. Thomas, H. Sindi, and G. M. Whitesides, "Simple Telemedicine for Developing Regions: Camera Phones and PaperBased Microfluidic Devices for Real-Time, Off-Site Diagnosis,” Anal. Chem., vol. 80, no. 10, pp. 3699-3707, May 2008.

[6] X. Li, D. R. Ballerini, and W. Shen, "A perspective on paper-based microfluidics: Current status and future trends,” Biomicrofluidics, vol. 6, no. 1, p. 011301, Mar. 2012.

[7] A. Nilghaz, L. Guan, W. Tan, and W. Shen, "Advances of Paper-Based Microfluidics for Diagnostics-The Original Motivation and Current Status,” ACS Sensors, vol. 1, no. 12, pp. 1382-1393, Dec. 2016.

[8] L. Li, X. Huang, W. Liu, and W. Shen, “Control Performance of Paper-Based Blood Analysis Devices through Paper Structure Design,” ACS Appl. Mater. Interfaces, vol. 6, no. 23, pp. 21624-21631, Dec. 2014. 


\section{WILEY-VCH}

[9] J. Su, M. Al-Tamimi, and G. Garnier, "Engineering paper as a substrate for blood typing bio-diagnostics,” Cellulose, vol. 19, no. 5, pp. 1749-1758, 2012.

[10] N. Afshari, P., Abolfathi, "A novel method for blood-typing using nitrocellulose," Biomed. Chromatogr., vol. 31, no. 7, 2016.

[11] J. Hansson, H. Yasuga, T. Haraldsson, and W. van der Wijngaart, "Synthetic microfluidic paper: high surface area and high porosity polymer micropillar arrays," Lab Chip, vol. 16, no. 2, pp. 298-304, 2016.

[12] D. R. Ballerini, X. Li, and W. Shen, "An inexpensive thread-based system for simple and rapid blood grouping,” Anal. Bioanal. Chem., vol. 399, no. 5, pp. 1869-1875, Feb. 2011.

[13] A. Nilghaz, D. R. Ballerini, L. Guan, L. Li, and W. Shen, "Red blood cell transport mechanisms in polyester thread-based blood typing devices," Anal. Bioanal. Chem., vol. 408, no. 5, pp. 1365-1371, Feb. 2016.

[14] A. Nilghaz, L. Zhang, M. Li, D. R. Ballerini, and W. Shen, "Understanding Thread Properties for Red Blood Cell Antigen Assays: Weak ABO Blood Typing,” Appl. Mater. interfaces, vol. 6, pp. 22209-22215, 2014.

[15] G. Basu, A. N. Roy, K. K. Satapathy, S. M. J. Abbas, L. Mishra, and R. Chakraborty, "Potentiality for value-added technical use of Indian sisal," Ind. Crops Prod., vol. 36, no. 1, pp. 33-40, 2012.

[16] E. Aracri, M. B. Roncero, and T. Vidal, "Studying the effects of laccase-catalysed grafting of ferulic acid on sisal pulp fibers,” Bioresour. Technol., vol. 102, no. 16, pp. 7555-7560, Aug. 2011.

[17] J. Casals-Terré, J. Farré-Lladós, A. Zuñiga, M. B. Roncero, and T. Vidal, "REPlicating RAPid Microfluidics: Self-Replicating Printer for Hydrophobic Pattern Deposition,” 3D Print. Addit. Manuf., vol. 4, no. 4, 2017.

[18] S. Gharehkhani, E. Sadeghinezhad, S. N. Kazi, H. Yarmand, A. Badarudin, M. R. Safaei, and M. N. M. Zubir, "Basic effects of pulp refining on fiber properties-A review,” Carbohydr. Polym., vol. 115, pp. 785-803, Jan. 2015.

[19] R. Alén, "Structure and Chemical composition of Wood.," in Chapter1. "In Forest products chemistry,” Peer Stenius Tappi Press, 2000, pp. 785-803.

[20] J. Higgins, H.G., Yong, "The beating Process: Primary effects and their influence on Pulp and Paper Propierties,” in The formation and structure of paper Transactions, Oxford British Paper and Board Makers’ Association, Ed. 1961, pp. 651-97.

[21] F. Carstens, J. A. F. Gamelas, and S. Schabel, “Engineering microfluidic papers: determination of fibre source and paper sheet properties and their influence on capillary-driven fluid flow," Cellulose, vol. 24, no. 1, pp. 295-309, 2017.

[22] P. Niskanen, K.; Kajanto, I.; Pakarinen, Papermaking Science and Technology. Tappi Press, 1998.

[23] M. Al-Tamimi, W. Shen, R. Zeineddine, H. Tran, and G. Garnier, "Validation of Paper-Based Assay for Rapid Blood Typing,” Anal. Chem., vol. 84, no. 3, pp. 16611668, Feb. 2012.

[24] M. S. Khan, G. Thouas, W. Shen, G. Whyte, and G. Garnier, "Paper Diagnostic for Instantaneous Blood Typing,” Anal. Chem., vol. 82, no. 10, pp. 4158-4164, May 2010.

[25] L. Guan, J. Tian, R. Cao, M. Li, Z. Cai, and W. Shen, "Barcode-Like Paper Sensor for Smartphone Diagnostics: An Application of Blood Typing,” Anal. Chem., vol. 86, no. 22, pp. 11362-11367, Nov. 2014.

[26] N. Yeow, H. McLiesh, and G. Garnier, “Indirect antiglobulin paper test for red blood cell antigen typing by flow-through method,” Anal. Methods, vol. 7, no. 11, pp. 46454649, May 2015.

[27] M. Li, J. Tian, R. Cao, L. Guan, and W. Shen, "A low-cost forward and reverse blood typing device—a blood sample is all you need to perform an assay," Anal. Methods, 


\section{WILEY-VCH}

vol. 7, no. 3, pp. 1186-1193, Jan. 2015.

[28] P. Jarujamrus, J. Tian, X. Li, A. Siripinyanond, J. Shiowatana, and W. Shen, "Mechanisms of red blood cells agglutination in antibody-treated paper," Analyst, vol. 137, no. 9, p. 2205, Apr. 2012.

Table I. Nomenclature and Measured paper properties

\begin{tabular}{|c|c|c|c|c|c|c|}
\hline $\begin{array}{c}\text { Paper } \\
\text { rev_BASIS WEIGHT }\end{array}$ & Refining (rev) & $\begin{array}{c}\text { Basis Weight } \\
\text { (g/m2) }\end{array}$ & $\begin{array}{c}\text { Average } \\
\text { Pore size } \\
(\mu \mathrm{m})\end{array}$ & $\begin{array}{c}\text { Thickness } \\
(\mu \mathrm{m})\end{array}$ & $\begin{array}{l}\text { Apparent } \\
\text { Density } \\
\text { (g/cm3) }\end{array}$ & $\begin{array}{c}\text { Capillary } \\
\text { rise } \\
(\mathrm{cm}) \\
\end{array}$ \\
\hline $0 \_50$ & 0 & $52.41 \pm 1.76$ & 23.3 & $145 \pm 12$ & $0.36 \pm 0.01$ & $15.6 \pm 0.35$ \\
\hline $0 \_100$ & 0 & $99.35 \pm 8.05$ & 20.1 & $303 \pm 4$ & $0.33 \pm 0.01$ & $15 \pm 0.40$ \\
\hline $2000 \_50$ & 2000 & $52.35 \pm 2.16$ & 17.6 & $108 \pm 4$ & $0.48 \pm 0.02$ & $9.3 \pm 0.15$ \\
\hline $2000 \_100$ & 2000 & $107.03 \pm 2.71$ & 8.7 & $205 \pm 9$ & $0.53 \pm 0.01$ & $8.8 \pm 0.20$ \\
\hline 600050 & 6000 & $50.04 \pm 1.37$ & 7.2 & $92 \pm 3$ & $0.58 \pm 0.02$ & $6.5 \pm 0.24$ \\
\hline Whatman 5 & ----- & $98.40 \pm 0.59$ & $2.5^{\star}$ & $186 \pm 1$ & $0.53 \pm 0.02$ & $4.05 \pm 0.22$ \\
\hline Whatman 40 & ----- & $88.18 \pm 0.58$ & $8^{*}$ & $192 \pm 2$ & $0.45 \pm 0.01$ & $6.05 \pm 019$ \\
\hline Whatman 1 & ----- & $86.35 \pm 0.63$ & $11^{*}$ & $165 \pm 1$ & $0.52 \pm 0.07$ & $6.23 \pm 0.26$ \\
\hline Whatman 41 & ----- & $85.76 \pm 0.40$ & $20^{*}$ & $205 \pm 6$ & $0.42 \pm 0.11$ & $9.6 \pm 0.20$ \\
\hline
\end{tabular}

* nominal particle retention size provided by the manufacturer

Table II. Paper properties

\begin{tabular}{cccccc}
\hline \multicolumn{6}{c}{ Fiber morphological properties of sisal papers refined at } \\
diferent degrees (rev)
\end{tabular}

Table II shows the values of ${ }^{\circ} \mathrm{SR}$, water retention value (WRV), fiber length, percentage of fines and width for each of the manufactured papers. 